\title{
Review
}

\section{Inhibiting Immune Checkpoints for the Treatment of Bladder Cancer}

\author{
S. Bidnur, R. Savdie and P.C. Black* \\ Vancouver Prostate Centre, University of British Columbia, Vancouver, B.C, Canada
}

\begin{abstract}
.
Background: Increasing evidence supporting the role of immune checkpoint blockade in cancer management has been bolstered by recent reports demonstrating significant and durable clinical responses across multiple tumour types, including metastatic urothelial carcinoma (mUC). The majority of these results are achieved via blockade of the programmed death (PD) axis, which like CTLA-4 blockade permits T-cell activation and immune-mediated anti-tumour activity- essentially harnessing the patient's own immune system to mount an anti-neoplastic response. However, while clinical responses can be striking, our understanding of the biology of immune checkpoint blockade is only beginning to shed light on how to maximize and even improve patient outcomes with immune checkpoint blockade, especially in UC.

Methods: We performed a literature review for immune checkpoint blockade with a focus on rationale for checkpoint therapy and outcomes in UC. We also highlight the advances made in other tumour types, with a focus on the recent 2015 meeting of the American Society for Clinical Oncology.

Results: In heavily pre-treated UC, trials are suggesting objective response rates above $30 \%$. These impressive results are seen across multiple different tumour types, especially those with high burden of DNA level mutations. Identification of prognostic biomarkers is currently under investigation, in order to improve patient selection. Interestingly, response to PD-1 directed therapy is seen even in patients with no evidence of PD-1 positivity on immunohistochemistry. This has led to the development of enhanced biomarkers including assessing DNA mutation rates and immune gene signatures, to improve patient selection.

Conclusions: Immune checkpoint blockade is an exciting cancer treatment modality which is demonstrating impressive clinical results across multiple tumour types. For UC, anti-PD directed therapy represents a much needed treatment in the metastatic, post chemotherapy context. Potential for these agents to have clinical utility in non-metastatic UC is still to be assessed.
\end{abstract}

Keywords: Bladder cancer, immune checkpoint blockade, PD-1/PD-L1, predictive biomarkers

\section{INTRODUCTION}

Cancer immunotherapy via immune checkpoint blockade has become an exciting and rapidly evolving subject in cancer management with new, targeted therapies demonstrating anti-neoplastic activity and survival benefits across multiple tumour types, with marked response rates in urothelial carcinoma (UC) of the bladder [1-4]. While cancer immunotherapy

\footnotetext{
*Correspondence to: Peter Black, Vancouver Prostate Centre University of British Columbia, Level 6, 2775 Laurel St, Vancouver, BC V5Z 1M9, Canada. Tel.: +1 604875 4301; E-mail: pblack@ mail.ubc.ca.
}

includes a broad range of therapeutic modalities, including anti-cancer vaccines, chimeric antigen receptors, and adoptive T-cell transfer, this review will focus on targeted therapies in cancer immunology, where monoclonal antibody blockade of co-signaling molecules required for proper T-cell activation and suppression can modulate the immune system to render an anti-neoplastic response.

There exists strong rationale for the use of these agents in genitourinary malignancies, especially in UC, which has already benefited from the use of immune-based treatments in the form of intravesical bacillus Calmette-Guerin (BCG) for over three 
decades. Immunotherapy with BCG is efficacious in non-muscle invasive bladder cancer (NMIBC), while enthusiasm for checkpoint blockade is fuelled by impressive responses in chemotherapy refractory metastatic UC (mUC). Immune checkpoint blockade thus represents a new pillar of UC treatment with the potential to improve outcomes across the spectrum disease. This review will highlight the biology of checkpoint blockade and focus on recent advances pertaining to the role of checkpoint inhibition in the treatment of UC.

\section{WHAT ARE CHECKPOINT INHIBITORS?}

The process whereby the immune system plays an active role in surveillance and elimination of cells undergoing malignant transformation has been long described. This process is referred to as immunoediting, and it is critical to the understanding of checkpoint inhibition [5]. Immuno-editing includes three key components: elimination, equilibrium and escape [5,6]. Cells having undergone malignant transformation as a result of accruing genomic mutations present tumour associated neoantigens (TAAs) on their cell surface via major histocompatibility complex class I (MHC-I), thereby allowing classical activation of the immune system as "non-self" and targeted cell death. In fact, increased T-cell cytolytic activity is associated with tumours bearing mutations in the antigen presenting machinery itself (i.e. mutations of the $\alpha$ and $\beta$ subunits of MHC-I) [7]. However, this process is dynamic and represents an equilibrium between the ability of the immune system to identify and eliminate malignant cells, and the ability of the malignant cells to escape such recognition. The mechanism of tumour cell (TC) escape may be due to decreases in neoantigen presentation, the establishment of a cytokine milieu that promotes an immunosuppressive tumour microenvironment (TME), or up-regulation of negative co-signals which prevent effective T-cell activation, thus allowing ongoing tumour growth [8]. Together, this process is described as the cancer-immunity cycle [9].

TCs interact with tumour associated immune cells (TAIC) within the TME to orchestrate successful immune escape [10]. Cell-mediated immunity employs inhibitory co-regulatory signaling in order to maintain self-tolerance, an evolutionary conserved mechanism of preventing damage to host tissues when the immune system is activated in response to tumour or infection [11]. Presently, most cancer immunotherapy strategies aim at restoring T-cellmediated anti-tumour activity, in essence harnessing the patient's own immune system for therapeutic benefit.

The three checkpoint molecules that have been studied most extensively and have become clinically important targets of drug therapy are cytotoxic T-lymphocyte associated protein 4 (CTLA-4), programmed cell death (PD)-1 and PD-ligand-1 (PD-L1). Ipilimumab (anti-CTLA-4), nivolumab and pembrolizumab (antiPD-1), and atezolizumab (anti-PD-L1) are the leading examples of a series of monoclonal antibodies under development that specifically block the inhibitory receptor-ligand interaction at the T-cell membrane. By inhibiting the immune checkpoint, these drugs permit activation of the immune response to TCs. Ipilimumab and nivolumab are now FDA-approved for clinical use in metastatic melanoma while atezolizumab has received FDA "breakthrough" status for non-small cell lung cancer (NSCLC) and UC of the bladder [12]. Pembrolizumab was recently granted a 'priority review' by the FDA for the treatment of NSCLC after progression on platinum-based chemotherapy, with a final decision pending in October 2015. There are many clinical trials ongoing currently that are testing these and many other checkpoint inhibitors.

CTLA-4 is expressed exclusively on T-cells and primarily regulates the amplitude of early $\mathrm{T}$-cell activation. While constitutively expressed on Foxp3 + regulatory T-cells $\left(\mathrm{T}_{\text {reg }}\right)$, CTLA-4 expression is induced in activated CD8 + effector $\mathrm{T}$ cells, where it induces down-regulation of $\mathrm{CD} 4+\mathrm{T}$ cell activity [13]. CTLA-4 counteracts the activity of the T cell co-stimulatory receptor CD28. Both compete for the same ligands on antigen presenting cells (APCs), CD80 and CD86, though CTLA-4 has a much higher affinity for both, thus dampening the CD28 stimulatory effect [14]. The critical role for CTLA-4 in silencing T cell activation was vividly demonstrated in the lethal systemic autoimmune sequelae observed in CTLA-4 knockout mice [15]. Indeed, targeting CTLA-4 (e.g. with ipilimumab) appears to carry the highest risks of autoimmune side effects when compared to PD-1 and PD-L1 toxicity $[16,17]$.

PD-1 is a cell-surface molecule that is activated by two ligands, PD-L1 and PD-L2. PD-L1 plays a more prominent role in lymphocyte regulation, being itself up-regulated by tumour-necrosis factor alpha (TNF- $\alpha$ ) and interferon-gamma(IFN- $\gamma$ ). PD-L1 is a $40-\mathrm{kDa}$ type 1 transmembrane protein that is known to suppress the immune system in cancer, pregnancy, tissue allografts and autoimmune diseases [18]. In cancer, 
PD-L1 expression occurs on both TCs, circulating immune cells and TAIC. PD-1 is expressed primarily on immune cells [19]. The primary role of PD-1/PD$\mathrm{L} 1$ interaction is to limit effector T-cell activity at the time of inflammatory responses to infection and to curb autoimmunity. PD-1 expression on lymphocytes is upregulated during T-cell activation [20]. PD-L1 binding results in inhibition of T-cell activation, proliferation and cytokine secretion. PD-1 is also highly expressed on the surface of $\mathrm{T}_{\text {regs }}$, but in this context PD-L1 binding enhances proliferation of this immunosuppressive phenotype [21]. The suppressive co-signal provided by PD-L1 and its relative PD-L2 upon binding to the PD-1 receptor renders a state of T-cell anergy, thereby promoting an immune tolerant TME [22]. TAICs recruited to this environment fail to manifest effector function despite abundance of antigen presentation on classical APCs and TCs themselves. Many tumours are highly infiltrated with $\mathrm{T}_{\text {reg }}$ cells that actively suppress effector immune response, and thus inhibition of the PD-1 pathway leads to decreased intra-tumoural infiltration and activity of $\mathrm{T}_{\text {reg }}$ cells [21].

Unlike CTLA-4, PD-1 is expressed on other non-T cell lymphocytes, including B-cells and natural killer cells (NK), where it limits the lytic activity of the latter [23]. Thus PD-1 blockade enhances NK cell activity and the production of antibodies by $\mathrm{PD} 1^{+} \mathrm{B}$-cells in addition to promoting effector $\mathrm{CD} 8+\mathrm{T}$-cell activation. Expression of PD-1 on CD8+ tumour infiltrating lymphocytes (TILs) may reflect T-cell anergy, a state of inactivation in which the cell remains primed to mount an immune response directed towards a tumour neoantigen but fails to manifest an immune response [24]. It appears TCs express PD-L1 in both an innate constitutive oncogenic pathway, via activation of the AKT and signal transducer and activator of transcription 3 (STAT3) pathways, and in an adaptive pathway. In the adaptive pathway PD-L1 is induced in the TC in response to presence of IFN- $\gamma$ in the TME [25]. Increasing understanding of the interactions within the TME is continuing to delineate the interplay of TCs, stromal cells and TILS and other TAICs in maintaining an immunosuppressive milieu [26].

\section{RATIONALE FOR IMMUNE CHECKPOINT BLOCKADE IN UROTHELIAL CARCINOMA}

Immune therapy has been established in bladder cancer for nearly four decades with the use of intravesical BCG for non-muscle invasive bladder cancer
(NMIBC) [27]. It is well recognized that BCG efficacy requires a competent immune system and functional T-cells subtypes amongst other immune machinery (NK cells, macrophage, granulocyte and dendritic cells in addition to various cytokines and interleukins), which are recruited to the bladder and found in large amounts in the urine following therapy [28-31]. Animal studies have confirmed the requirement of both CD4 + and CD8 + TILs, with depletion of either resulting in loss of BCG-mediated antitumour activity [32]. The exact mechanism by which BCG orchestrates a favorable immune-mediated anti-tumour response is not fully elucidated. Considering that BCG is instilled intravesically when tumour burden is lowest, its mechanism is almost certainly multifactorial, via immune modulation, direct interactions with TAICs, and likely also interactions with benign and malignant urothelial cells [33].

One of the first studies on checkpoint molecules in UC investigated PD-L1 expression in 280 patients with a broad spectrum of stages and grades [34]. This study demonstrated increased PD-L1 positivity (defined as $>1 \%$ positive cells on immunohistochemistry (IHC)) with higher stage and grade, although the highest rate of PD-L1 positivity was observed in carcinoma in situ (CIS). Interestingly, the most diffuse (>90\%) and intense PD-L1 staining was seen in BCG-granulomas in 11 of the 16 patients with persistent disease post BCG, while only three of these patients had PD-L1 positive tumours prior to BCG therapy. While this study by Inman et al. focused on PD-L1 expression in UC cells, another report by Nakanishi et al. highlighted the correlation between high PD-L1 expression in TAICs and poor clinical outcomes, including recurrence and overall survival (OS) [35]. On multivariate analysis, TAIC PD-L1 expression was a more significant prognostic factor than WHO grade. A study by Boorjian et al. correlated tumour cell PD-L1 status with advanced disease and decreased OS in patients undergoing radical cystectomy for organ confined UC. A more recent study evaluating 302 patients has shown a similar association between PD-L1 status and increased risk of death for patients with organ-confined disease after cystectomy $(P=0.02)[36,37]$. Recently, Bellmunt et al. were the first to describe TAIC PD-L1 expression associated with improved OS in patients with metastatic platinumrefractory UC [38]. No association was found with TC PD-L1 expression and OS. The retrospective nature of this study makes it susceptible to confounding.

The potential role of cancer neoantigens in immune regulation of cancer suggests that UC may be particularly suitable for checkpoint blockade. The mutational 
landscape that results from chronic carcinogen exposure, typical of melanoma (ultraviolet light), NSCLC and UC (cigarette smoking), results in multiple protein-level errors resulting in a high level of malignancy associated neo-antigen presentation within the TME [39, 40]. This should result in T-cell activation against these "non-self" peptides. Higher exon mutation rate in NSCLC has been shown to correlate to response to anti-PD-1 therapy, implying maximal benefit of checkpoint blockade occurs when there is a higher level of background mutation [41]. In UC, a similarly high non-synonymous mutation rate has been reported [39]. Cazier et al. reported a mean of 219 potential functional mutation changes in a series of UC specimens from a range of stages and grades [42]. Interestingly, the non-synonymous mutations for patients with UC was highest in those patients with urothelial CIS, where it reached 561.

The latest report on PD-L1 expression related to BCG therapy was reported recently at the 2015 annual meeting of the American Society of Clinical Oncology (ASCO) [43]. A cohort of patients who had undergone two transurethral bladder tumour resections (TURBT) that were separated by at least three months was identified, and the tumours were stained by IHC for PD-L1 expression. Any degree of staining was considered positive. Of these patients, 23 had received intravesical BCG between the first and second TURBT. In this cohort, PD-L1 expression was found more commonly in the second sample (7/39 vs. 12/39) but there was no correlation to BCG therapy. These preliminary findings require further study in larger cohorts.

Baras et al. recently demonstrated the correlation of PD-L1 staining by IHC in cisplatin-based neo-adjuvant chemotherapy (cNAC) responders and non-responders [44]. They concluded that cNAC non-responders frequently exhibit PD-L1 tumoural staining (40\%), and suggested that these could be candidates for anti-PDL1 therapy. NCT02451423 is examining this in a phase II trial (Table 1). On the other hand, Choi et al. noted on gene expression microarray that cisplatin-sensitive basal tumours were enriched for an immune signature consistent with immune infiltration [45]. This signature included CTLA-4 and CD8 but not PD-L1.

One of first clinical trials evaluating immunotherapy in UC was performed by Carthon et al. [46]. The study evaluated the safety and immunomodulatory effects of two pre-operative anti-CTLA-4 doses prior to radical cystectomy in a small cohort of 12 patients, half treated at $3 \mathrm{mg} / \mathrm{kg} / \mathrm{dose}$ and half at $10 \mathrm{mg} / \mathrm{kg} / \mathrm{dose}$. While most adverse events (AE) were low grade, severe diarrhea and ischemic papillopathy resulted in delay of surgery.
Importantly, this study correlated changes in TAIC from surgical specimens with changes in T-cell expression markers from peripheral blood, suggesting that the latter may be used to monitor and assess effect of checkpoint blockade.

\section{CLINICAL TRIALS WITH PD-1/PD-L1 INHIBITORS IN UC}

The FDA granted the anti-PD-L1 antibody atezolizumab "breakthrough" status in bladder cancer in 2014 based on encouraging results from a phase Ia expansion trial with adaptive design that was reported in 2014 (with minimum 6 week follow-up) [47] and updated recently at ASCO 2015 (with minimum 12 week follow-up). The vast majority of patients $(92.5 \%)$ in this trial had failed prior platinum-based chemotherapy for mUC of the urethra, bladder or upper tract. Initially only patients with IHC evidence of PD-L1 expression were enrolled, but after surpassing futility criteria, the trial was expanded to include patients regardless of PD-L1 expression. PD-L1 was scored as $0,1,2$, and 3 , corresponding to staining of $<1 \%$, $1-5 \%, 5-10 \%$ and $>10 \%$. Overall 205 patients were screened, of whom $23 \%$ were considered positive for PD-L1 (IHC score 2-3) on TAICs, and 4\% were PDL1 positive on the TCs themselves. Importantly, PD-L1 expression in the TAICs but not in the TCs correlated to response in the 67 patients considered evaluable for efficacy. In the updated results, median OS was between 10 and 14 months, and an objective response (RECIST criteria) was observed in $46 \%$ of PD-L1 positive patients compared to $16 \%$ of PD-L1 negative patients [48].

Early results for pembrolizumab in the second-line setting in 29 evaluable patients with advanced urothelial carcinoma (any location, 66\% metastatic) were reported at ASCO 2015. Overall response rate was $27.6 \%$ (11\% complete, $16 \%$ partial) with median duration of response not reached at 13 months median follow up [49]. OS in this platinum refractory cohort was 12.7 months.

These early results have generated a storm of enthusiasm and activity for testing these two agents and other checkpoint inhibitors in UC patients in multiple different clinical scenarios. A snapshot of current activity summarizing trials listed on clinicaltrials.gov is provided in Table 1, but many other trial concepts are also being considered. A large multicenter phase III trial with atezolizumab that aims to recruit 767 patients with locally advanced or mUC who have failed prior 


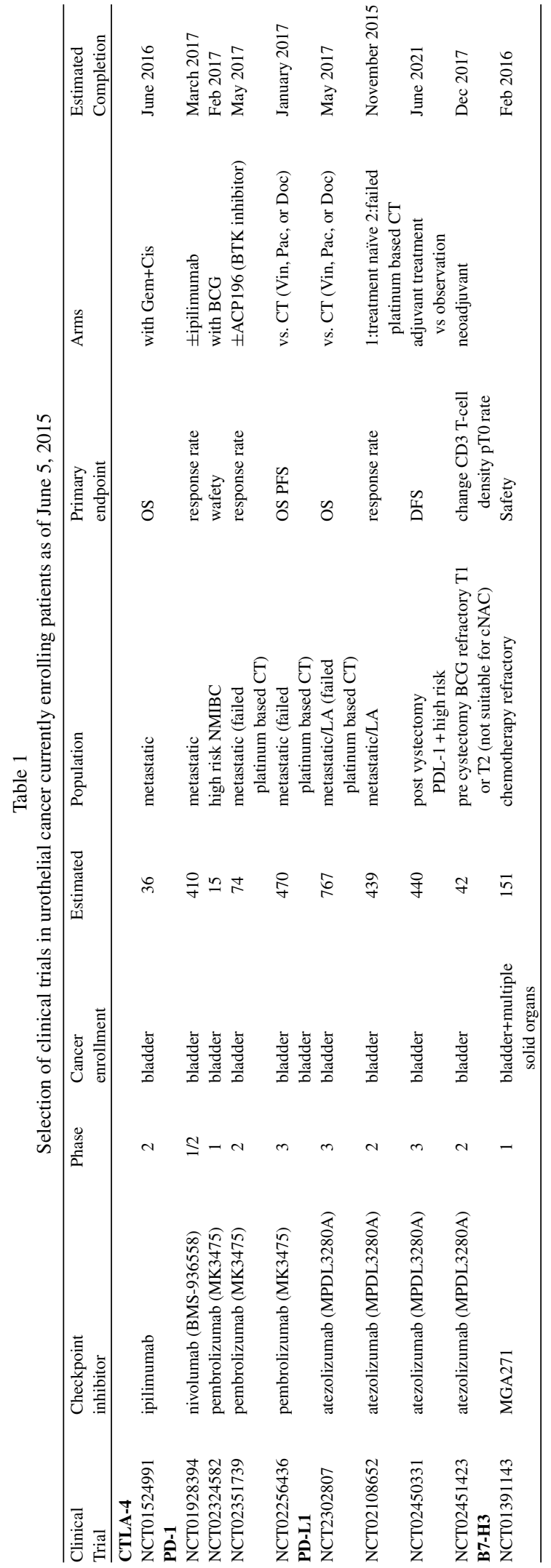

platinum-based chemotherapy is currently underway (NCT02302807).

Harnessing the rational combination of immunostimulatory BCG therapy with inhibition of immunosuppressive targets such as the PD-1 axis is being studies across several clinical trials currently. NCT02324582 is a phase I interventional study assessing combination PD-1 blockade with BCG therapy for HGT1 bladder cancer, while NCT01838200 combines these two agents in advanced stage melanoma, with intralesional BCG injection. These results could have major implications in bladder cancer, where combination therapy to maximize the efficacy of the current standard of care BCG could markedly improve outcomes in patients with high-risk non-muscle invasive disease. More nuanced combination regimens with BCG and checkpoint inhibitors, including combination regimes that follow an induction and maintenance course have yet to be described and will likely be shaped by the results of the aforementioned trials.

\section{PREDICTIVE BIOMARKERS}

As with most novel targeted therapies, there is an intense research focus on identifying predictive markers that will aid in selection the patients most likely to respond to checkpoint blockade. The early experience in UC is mirrored in other organ sites, where tissue expression of PD-L1 by IHC predicts increased treatment response [50]. It is increasingly recognized that specifying IHC positivity by cell type within tumour tissue (i.e. TC vs TAIC subtypes vs stroma) may increase the predictive power of PD-1/PD-L1 staining (discussed below). However, predicting response goes beyond PD-L1 IHC, as even some patients with no PD-L1 positivity anywhere in the tumour specimen respond to therapy, and thus additional novel predictive markers are under investigation (Table 2).

A major limitation for the use of PD-1/PD-L1 status as a biomarker has been the heterogeneity of reported methods for assessing PD- positivity on IHC. Different drug manufacturers have developed multiple different antibodies, each with their respective proprietary IHC assay as a companion diagnostic. The antibodies are different in their characteristics and operating stability, which likely affects inter-study comparisons. The location of positive PD-L1 staining is generally classified as TC versus TAIC, although tissue stromal staining has also been described by some investigators, and a standardized classification scheme has not be established. The majority of studies consider strong PD-L1 


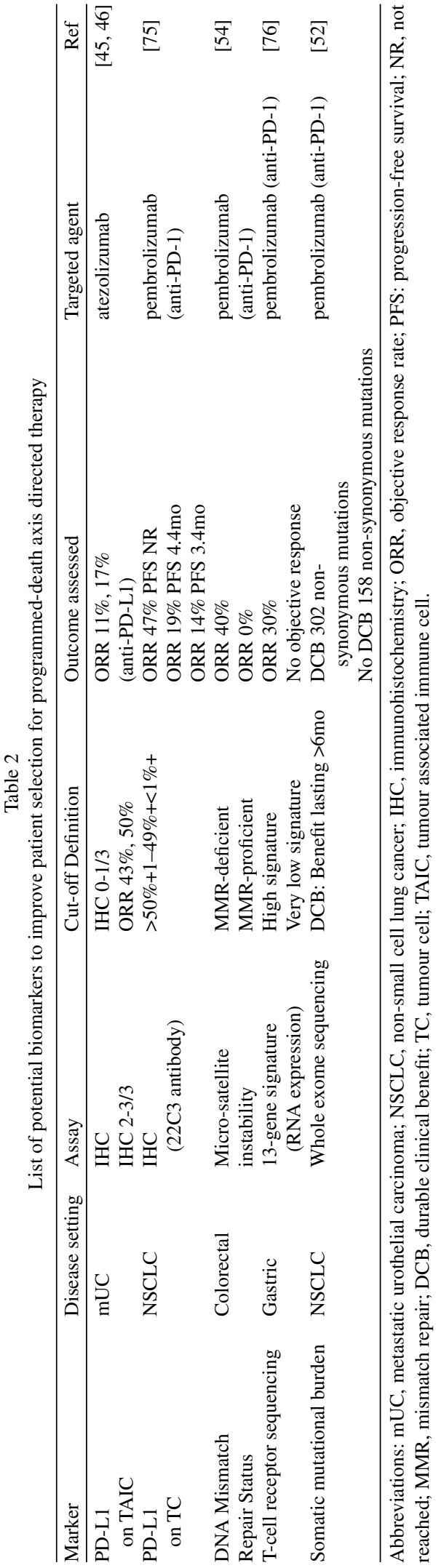

staining of $>5 \%$ (IHC staining scored 3/3) on either TCs or TAICs as positive, while other papers consider $1 \%$ as a positive cut-off. An immediate challenge in this field of research regardless of the cancer type is to establish standard criteria for assessing PD-L1 expression in order to improve patient selection.

The potential pitfalls of IHC are exemplified in the patients with PD-L1 positive triple negative breast cancer enrolled in the Keynote-012 phase Ib study [51]. PD-L1 positivity was defined as $\geq 1 \%$ on either TCs or TAIC. Objective response rate (ORR) was similar in tumours with only TC PD-L1 positivity (33\%) versus both TC and TAIC positivity (29\%). However, a 9\% ORR was still seen in the TC PD-L1 negative group. However, when TCs and TAICs were both PD-L1 negative, there was a $0 \%$ ORR. The authors concluded that specifically assessing both TCs and TAICs for positivity may improve patient selection. The significance of differentiating between TC positive and TAIC positive staining was highlighted in NSCLC studies at ASCO 2015. While strong PD-L1 staining (IHC 3/3) on either TCs or TAIC was associated with $44 \%$ ORR versus $16 \%$ in the weak or no staining group, tumour histology differed significantly between the IHC $3 / 3 \mathrm{TC}$ vs TAIC positive groups. The former was associated with a TME with minimal TAIC presence, while the latter is associated with strong TAIC presence at the tumourstromal interface [52]. Further studies are needed to analyze the TME histology and PD-L1 positivity status in order to guide patient selection.

With few other options available for patients who fail platinum-based chemotherapy for advanced UC, and even fewer for those who are cisplatin-ineligible, it is difficult to deny patients drugs like atezolizumab with a 14\% ORR in PD-L1 IHC 0/1 status based on experimental, not yet established biomarkers, especially when this ORR is often prolonged and can be achieved with minimal toxicity Therefore, development and validation of enhanced predictive biomarkers, beyond tumour PD-1 and PD-L1 status are being pursued.

Neoantigen burden has been identified as a marker of response to immune-targeting agents, as described above [53]. Mutational load correlated to clinical response in NSCLC treated with PD-1 blockade and melanoma treated with CTLA-4 blockade $[54,55]$. UC is known to have one of the highest burdens of somatic mutations, which should translate into high neoantigen load. Somatic mutations can easily be quantified with whole exome sequencing of tumour tissue. The vast majority of mutations, however, do not result in formation of neoantigens that are recognized by T-cells. Methods and algorithms are being developed to use 
sequencing data along with HLA haplotyping from normal cells to estimate neoantigen load [56].

Building on this same concept, Le et al. recently reported that deficiency in DNA mismatch repair (MMR) is highly correlated to response to pembrolizumab in colorectal cancer (CRC) and other solid tumours (UC was not included) [57]. The ORR was 40\% (4/10) for MMR-deficient CRC, $0 \%$ (0/18) for MMR-proficient CRC and 71\% (5/7) for MMR-deficient non-CRC. Whole-exome sequencing revealed a mean of 1782 somatic mutations per tumour in MMR-deficient tumours, as compared with 73 in MMR-proficient tumours, and high somatic mutation loads were associated with prolonged progression-free survival. MMR status is determined by analysis of microsatellite stability. It will be important to correlate this marker to clinical outcome in UC.

\section{TOXICITY AND TOLERABILITY OF IMMUNE CHECKPOINT BLOCKADE THERAPIES}

Most studies investigating blockade of PD-1 or PDL1 report favorable toxicity profiles with the vast majority of treatment related AE being of low grade $[3,47]$. Various doses of anti-PD-L1 therapy ranging from 0.3 to $10 \mathrm{mg} / \mathrm{kg}$ administered over a median of 12 weeks, resulted in an overall $9 \%$ rate of grade 3 or $4 \mathrm{AE}$ treated managed with treatment interruption or discontinuation. While $91 \%$ of patients reported any grade $\mathrm{AE}$, most were self-limiting including fatigue, rash, diarrhea, arthralgia, nausea, pruritis and headache. Drug infusion-related AEs were reported only in the $10 \mathrm{mg} / \mathrm{kg}$ study arm and were treated with reduced infusion rate, prophylactic anti-histamines and antipyretics. In the phase Ib study in UC described above by Powles et al. reviewed in section three, PDL1 inhibitory therapy was well tolerated, with $4.4 \%$ patients experiencing grade 3 adverse events, namely thrombocytopenia, decreased blood phosphorus, and asthenia. Of the 53\% who experienced grades 1-2 AEs, the majority were decreased appetite, nausea, and fatigue. No grade 4 or 5 treatment related AEs were reported. This toxicity profile is in contrast to reported tolerability of CTLA-4 blockade, which appears to be associated with higher grade AE characterized predominantly by various grades of dermatitis and colitis [58].

The recently reported phase III randomized study of combination nivolumab plus ipilimuab versus ipilimumab alone in untreated metastatic melanoma sheds increased light on the nature of $\mathrm{AE}$ with checkpoint inhibitors (clinical results discussed below) [59]. Grade III and IV AE, predominantly colitis and diarrhea, were more common in the combination group versus the ipilimumab alone group (54\% vs $24 \%$, respectively). These AE occurred early in the combination arm, and decreased during the nivolumab only maintenance phase.

\section{LESSONS LEARNED FROM CHECKPOINT INHIBITORS IN OTHER ORGAN SITES}

Checkpoint blockade for UC is not happening in isolation, but instead is one piece in a global deluge of clinical trials with inhibitors of CTLA-4, PD-1 and PDL1, with additional new immune targets on the horizon. Objective responses measured with RECISTv1.1 criteria have been reported in subsets of patients with melanoma, NSCLC, gastric cancer, ovarian cancer, head and neck squamous cell cancer (HNSCC), and renal cell carcinoma (RCC). Early results in prostate cancer have not been as promising [60]. Progress in these other disease sites sets the stage for ongoing and future development in UC, as highlighted in some examples below.

\section{Melanoma - Combination immunotherapy more efficacious than monotherapy}

Successful phase III trials of ipilimumab led to its approval by the FDA for metastatic melanoma in 2014. Since then the focus has moved to combination therapy with anti-PD-1 antibodies, after pre-clinical data suggested synergistic efficacy between these drugs [61]. A recently reported landmark phase III trial of firstline nivolumab plus ipilimumab versus ipilimumab alone showed a $61 \%$ ORR for the combination compared to $11 \%$ for ipilimumab alone [59]. $22 \%$ of patients in the combination arm experienced a complete response. Interestingly, a more recent phase III trial also investigating combination nivolumab with ipilimumab found similar results, but in patients with PD-L1 + tumours, progression-free survival (PFS) was the same in the nivolumab alone and combination arms (14 months) [62]. Patients with PD-L1- tumours experienced more benefit from combination therapy, 11.2 vs 5.3 months. This highlights the potential of combination immunotherapy to maximize clinical response, especially with appropriately selected patients. 
Non-small cell lung carcinoma (NSCLC) - Moving checkpoint blockade into first line therapy for metastatic NSCLC

Multiple clinical trials are currently investigating the efficacy of PD-1 and PD-L1 inhibition in NSCLC, including as first line therapy against standard of care, and incorporation of PD-L1 positivity as a biomarker. Checkmate-017 was a randomized phase III trial comparing nivolumab to docetaxel for metastatic NSCLC [63]. This study was stopped early due to superior OS seen in the nivolumab arm. Other trials include Checkmate 057 and 026 , which are also randomized phase III studies investigating nivolumab as $2 \mathrm{nd}$ or $3 \mathrm{rd}$ line treatment in non-squamous NSCLC versus docetaxel (fully accrued), and as 1st line in PD-L1 positive NSCLC, respectively. Keynote-024 and Keynote-042 are both recruiting patients with metastatic NSCLC, with comparisons between pembrolizumab and standard of care chemotherapy as first line treatment. In both phase III studies, patients must have evidence of tumour PD-L1 positivity for outcomes measurement based on PD-L1 status.

The recently presented phase II POPLAR clinical trial demonstrated that treatment with atezolizumab (MPDL3280A) doubled OS compared with docetaxel as second line therapy in patients with PD-L1positive NSCLC [64]. Results from the phase II trial FIR showed comparable response rates compared to conventional chemotherapy in both chemo-na'ive and previously treated patients. Furthermore, PDL-1 expression correlated positively with response rates. These agents appear ready for testing in the first line setting [63].

\section{Breast - Immunophenotypic parallels between triple negative breast cancer and basal subtype $U C$}

Significant molecular similarities between breast and bladder cancer have become apparent in the past 18 months, including especially the recapitulation in UC of the luminal and basal subtypes familiar to us from breast cancer [ $40,45,65]$. These similarities raise the possibility that new insights in breast cancer may suggest directions for investigation in UC. Both PD-1 + TILs and PD-L1 + carcinoma cells are seen more often in triple negative breast carcinoma, which corresponds molecularly to the basal subtype of UC, than in other breast cancer subtypes [66]. Clinical evaluation of checkpoint blockade has focused on triple negative metastatic disease. In this subset of breast cancer patients, objective responses have been reported in $18.5 \%$ of patients with pembrolizumab [51]. It remains to be demonstrated whether UC subtypes are predictive of response to checkpoint blockade.

\section{Renal cell carcinoma (RCC) - Targeting the PD-1 axis is effective second line treatment, not only following chemotherapy}

Like bladder cancer, there is a precedent for using immunotherapies in RCC, although systemic interferon and interleukin-2 therapies have been mostly abandoned with the advent of novel targeted therapies like vascular endothelial growth factor (VEGF)- and tyrosine kinase inhibitors (TKI) [67]. The use of PD-1 directed therapy in the setting of TKI-refractory disease highlights the utility of these agents as effective therapy in treatment-refractory disease. Multiple trials have now been reported on checkpoint inhibitors in metastatic RCC (mRCC). Nivolumab (anti-PD-1) is currently the lead compound in mRCC. An ORR of $20 \%$ was observed in a phase II trial in 168 patients, of whom $70 \%$ had undergone previous systemic therapy. As noted in many studies testing checkpoint inhibitors, many patients with a response demonstrated durable responses. The median overall survival varied from 18.2 to 25 months depending on drug dose, which was longer than the rates described in other studies in TKI-refractory metastatic RCC studies using newer generation TKIs such as axitinib [68].

\section{FUTURE DIRECTIONS}

Many trials have shown dismal results of second line chemotherapy and targeted therapies in $\mathrm{mUC}$, with ORRs in the range of approximately $12 \%$ [69-73]. However, early phase trials of checkpoint blockade in the same patients have demonstrated ORRs between $28 \%$ and $34 \%$ [48, 49]. Similar major advances are being observed across multiple heavily pre-treated tumour types, thus driving enthusiasm for further evaluation of these agents in multiple phase III trials. The rapid evolution and strong clinical results of checkpoint blockade are supported by translational research that supports the biology of T-cell co-stimulatory blockade for cancer treatment. Inhibition of CTLA-4 and the PD-1/PD-L1 axis is just the beginning, as inhibitors of other checkpoint molecules are under development. The potential application of checkpoint blockade to earlier disease states including BCGunresponsive high grade NMIBC has generated a lot of excitement in the bladder cancer community [43]. 
For this treatment modality to advance, results of favourable phase III clinical trials must also be accompanied by improvements in patient selection and biomarker development. It is important to highlight that the impressive clinical results cited at recent meetings may be even more pronounced with more precise patient selection. Improved patient selection is also important for pharmacoeconomic analyses. Although prices are certain to change moving forward, cost estimates for anti-PD-L1 directed therapy have reached as much as one million dollars per patient per year [12].

With the rapid expansion of cancer immunotherapy, many new potential checkpoint pathways have been identified that appear to have a similar suppressive effect on TAIC and are under investigation in multiple tumour types [11]. These include HLA-G, CD73, LAG-3 and B7-H3, with clinical trials underway to investigate whether these new checkpoints may offer similar clinical results as PD-1/PD-L1 targeted therapy [74]. Future research will identify not only new potential checkpoints but will also assess combination checkpoint blockade. In UC, the potential for combination and/or sequencing with intravesical BCG is a novel treatment paradigm that will require investigation.

Overall, the rapid development of new targeted therapies against T-cell co-regulatory molecules has increased our understanding of tumour biology, particularly the complexities of the TME. The result has been significant improvements in ORR and OS across multiple tumour types. For UC, these advances represent new pillars of treatment, especially in $\mathrm{mUC}$ where new therapies have been lacking.

\section{REFERENCES}

[1] Hodi FS, et al. Improved survival with ipilimumab in patients with metastatic melanoma. N Engl J Med 2010;363:711-23.

[2] Robert C, et al. Ipilimumab plus dacarbazine for previously untreated metastatic melanoma. N Engl J Med 2011; 364:2517-26.

[3] Brahmer JR, et al. Safety and activity of anti-PD-L1 antibody in patients with advanced cancer. N Engl J Med 2012; 366:2455-65

[4] Topalian SL, et al. Safety, activity, and immune correlates of anti-PD-1 antibody in cancer. N Engl J Med 2012;366:244354.

[5] Tomihara K, Curiel TJ, Zhang B. Optimization of immunotherapy in elderly cancer patients. Crit Rev Oncog 2013;18:573-83.

[6] Schreiber RD, Old LJ, Smyth MJ. Cancer immunoediting: Integrating immunity's roles in cancer suppression and promotion. Science 2011;331:1565-70.

[7] Rooney MS, Shukla SA, Wu CJ, Getz G., Hacohen N. Molecular and genetic properties of tumors associated with local immune cytolytic activity. Cell 2015;160:48-61.
[8] Dong H, et al. Tumor-associated B7-H1 promotes T-cell apoptosis: A potential mechanism of immune evasion. Nat Med 2002;8:793-800.

[9] Chen DS, Mellman I. Oncology meets immunology: The cancer-immunity cycle. Immunity 2013;39:1-10.

[10] Hanahan D, Weinberg RA. Hallmarks of cancer: The next generation. Cell 2011;144:646-74.

[11] Pardoll DM. T he blockade of immune checkpoints in cancer immunotherapy. Nat Rev Cancer 2012;12: 252-64.

[12] Carosella ED, Ploussard G, LeMaoult J, Desgrandchamps F. A Systematic Review of Immunotherapy in Urologic Cancer: Evolving Roles for Targeting of CTLA-4, PD-1/PD-L1, and HLA-G. Eur Urol 2015;68:267-79.

[13] Lenschow DJ, Walunas TL, Bluestone JA. CD28/B7 SYSTEM OF T CELL COSTIMULATION. Annual Review of Immunology 1996;14:233-58.

[14] Rudd CE, Taylor A., Schneider H. CD28 and CTLA-4 coreceptor expression and signal transduction. Immunol Rev 2009;229:12-26.

[15] Tivol EA, et al. CTLA4Ig prevents lymphoproliferation and fatal multiorgan tissue destruction in CTLA-4-deficient mice. J Immunol 1997;158:5091-4

[16] Ernst B, Anderson KS. Immunotherapy for the treatment of breast cancer. Curr Oncol Rep 2015;17:5.

[17] Simeone E, Ascierto PA. Immunomodulating antibodies in the treatment of metastatic melanoma: The experience with anti-CTLA-4, anti-CD137, and anti-PD1. J Immunotoxicol 2012;9:241-7.

[18] Freeman GJ, et al. Engagement of the PD-1 immunoinhibitory receptor by a novel B7 family member leads to negative regulation of lymphocyte activation. J Exp Med 2000; 192:1027-34.

[19] Ohaegbulam KC, Assal A, Lazar-Molnar E, Yao Y, Zang X. Human cancer immunotherapy with antibodies to the PD-1 and PD-L1 pathway. Trends Mol Med 2015;21:24-33.

[20] Ishida Y, Agata Y, Shibahara K., Honjo T. Induced expression of PD-1, a novel member of the immunoglobulin gene superfamily, upon programmed cell death. EMBO J 1992;11:3887-95.

[21] Francisco LM, et al. PD-L1 regulates the development, maintenance, and function of induced regulatory $\mathrm{T}$ cells. J Exp Med 2009;206:3015-29.

[22] Tsushima F, et al. Interaction between B7-H1 and PD-1 determines initiation and reversal of T-cell anergy. Blood 2007;110:180-5

[23] Fanoni D, et al. New monoclonal antibodies against B-cell antigens: Possible new strategies for diagnosis of primary cutaneous B-cell lymphomas. Immunol Lett 2011;134: 157-60.

[24] Ahmadzadeh M, et al. Tumor antigen-specific CD8 T cells infiltrating the tumor express high levels of PD-1 and are functionally impaired. Blood 2009;114:1537-44.

[25] Wilke CM, et al. Dual biological effects of the cytokines interleukin-10 and interferon-gamma. Cancer Immunol Immunother 2011;60:1529-41.

[26] Mellman I, Coukos G, Dranoff G. Cancer immunotherapy comes of age. Nature 2011;480:480-9.

[27] Morales A, Eidinger D, Bruce AW. Intracavitary Bacillus Calmette-Guerin in the treatment of superficial bladder tumors. J Urol 1976;116:180-3.

[28] De Boer EC, et al. Presence of activated lymphocytes in the urine of patients with superficial bladder cancer after intravesical immunotherapy with bacillus Calmette-Guerin. Cancer Immunol Immunother 1991;33:411-6. 
[29] Eto M, et al. Importance of urinary interleukin-18 in intravesical immunotherapy with bacillus calmette-guerin for superficial bladder tumors. Urol Int 2005;75:114-8.

[30] Jackson AM, et al. Changes in urinary cytokines and soluble intercellular adhesion molecule-1 (ICAM-1) in bladder cancer patients after bacillus Calmette-Guerin (BCG) immunotherapy. Clin Exp Immunol 1995;99:369-75.

[31] Morton D, Eilber FR, Malmgren RA, Wood WC. Immunological factors which influence response to immunotherapy in malignant melanoma. Surgery 1970;68:158-63; discussion 163-4.

[32] Ratliff TL, Ritchey JK, Yuan JJ, Andriole GL, Catalona WJ. T-cell subsets required for intravesical BCG immunotherapy for bladder cancer. J Urol 1993;150:1018-23.

[33] Redelman-Sidi G, Glickman MS, Bochner BH. The mechanism of action of BCG therapy for bladder cancer-a current perspective. Nat Rev Urol 2014;11:153-62.

[34] Inman BA, et al. PD-L1 (B7-H1) expression by urothelial carcinoma of the bladder and BCG-induced granulomata: Associations with localized stage progression. Cancer 2007;109:1499-505.

[35] Nakanishi J, et al. Overexpression of B7-H1 (PD-L1) significantly associates with tumor grade and postoperative prognosis in human urothelial cancers. Cancer Immunol Immunother 2007;56:1173-82.

[36] Boorjian SA, et al. T-cell coregulatory molecule expression in urothelial cell carcinoma: Clinicopathologic correlations and association with survival. Clin Cancer Res 2008;14: 4800-8.

[37] Xylinas E, et al. Association of T-cell co-regulatory protein expression with clinical outcomes following radical cystectomy for urothelial carcinoma of the bladder. Eur J Surg Oncol 2014;40:121-7.

[38] Bellmunt $\mathbf{J}$, et al. Association of PD-L1 expression on tumor-infiltrating mononuclear cells and overall survival in patients with urothelial carcinoma. Ann Oncol 2015;26: 812-7.

[39] Kandoth C, et al. Mutational landscape and significance across 12 major cancer types. Nature 2013;502: 333-9.

[40] Cancer Genome Atlas Research N. Comprehensive molecular characterization of urothelial bladder carcinoma. Nature 2014;507:315-22.

[41] Rizvi NA, et al. Mutational landscape determines sensitivity to PD-1 blockade in non-small cell lung cancer. Science (2015).

[42] Cazier JB, et al. Whole-genome sequencing of bladder cancers reveals somatic CDKN1A mutations and clinicopathological associations with mutation burden. Nat Commun 2014;5:3756.

[43] Hurwitz MA, Yao A, Hafez X, Schalper N, Rimm K, Petrylak DD. The effect of BCG intravesical therapy and recurrence on PDL1 expression in non-invasive bladder cancers. J Clin Oncol 2015;33:e15504.

[44] Baras AAM, Guner M, Gandhi G, Liu N, Taube J, Bivalacqua J, Netto TG. PDL1 status in muscle invasive urothelial bladder carcinoma (MIBC) in the context of neoadjuvant cisplatin based chemotherapy. J Urol 2015;193:e703.

[45] Choi W, et al. Identification of distinct basal and luminal subtypes of muscle-invasive bladder cancer with different sensitivities to frontline chemotherapy. Cancer Cell 2014;25:152-65.

[46] Carthon BC, et al. Preoperative CTLA-4 blockade: Tolerability and immune monitoring in the setting of a presurgical clinical trial. Clin Cancer Res 2010;16:2861-71.
[47] Powles T, et al. MPDL3280A (anti-PD-L1) treatment leads to clinical activity in metastatic bladder cancer. Nature 2014;515:558-62.

[48] Petrylak D, et al. A phase Ia study of MPDL3280A (antiPDL1): Updated response and survival data in urothelial bladder cancer (UBC). J Clin Oncol 2015;33(15_suppl):4501.

[49] Plimack E, et al. Pembrolizumab (MK-3475) for advanced urothelial cancer: Updated results and biomarker analysis from KEYNOTE-012. J Clin Oncol 2015;33(suppl); abstr 4502.

[50] Herbst RS, et al. Predictive correlates of response to the anti-PD-L1 antibody MPDL3280A in cancer patients. Nature 2014;515:563-7.

[51] Buisseret L, Specht J, Dees EC, Berger R, Gupta S, Geva R, Pusztai L, Gause CK, Karantza V, Nanda R. KEYNOTE-012: A phase Ib study of pembrolizumab (MK-3475) in patients (pts) with metastatic triple-negative breast cancer (mTNBC). Ann Oncol 2015;26:6.

[52] Gettinger S, et al. Molecular, immune and histopathological characterization of NSCLC based on PDL1 expression on tumor and immune cells and association with response to the anti-PDL1 antibody MPDL3280A. J Clin Oncol 2015;33(suppl); abstr 3015.

[53] Schumacher TN, Schreiber RD. Neoantigens in cancer immunotherapy. Science 2015;348:69-74.

[54] Rizvi NA, et al. Cancer immunology. Mutational landscape determines sensitivity to PD-1 blockade in non-small cell lung cancer. Science 2015;348:124-8.

[55] Snyder A, et al. Genetic basis for clinical response to CTLA-4 blockade in melanoma. N Engl J Med 2014;371:2189-99.

[56] Rajasagi M, et al. Systematic identification of personal tumorspecific neoantigens in chronic lymphocytic leukemia. Blood 2014; 124:453-62.

[57] Le DT, et al. PD-1 Blockade in Tumors with Mismatch-Repair Deficiency. N Engl J Med 2015;372:2509-20.

[58] Weber JS, Kahler KC, Hauschild A. Management of immune-related adverse events and kinetics of response with ipilimumab. J Clin Oncol 2012;30:2691-7.

[59] Postow MA, et al. Nivolumab and Ipilimumab versus Ipilimumab in Untreated Melanoma. New England Journal of Medicine 2015;372:2006-17.

[60] Kantoff PW, et al. Sipuleucel-T immunotherapy for castration-resistant prostate cancer. N Engl J Med 2010; 363:411-22.

[61] Nakamoto N, et al. Synergistic reversal of intrahepatic HCVspecific CD8 T cell exhaustion by combined PD-1/CTLA-4 blockade. PLoS Pathog 2009;5:e1000313.

[62] Larkin J, et al. Combined Nivolumab and Ipilimumab or Monotherapy in Untreated Melanoma. N Engl J Med 2015;373:23-34.

[63] Spigel D, et al. Clinical activity and safety from a phase II study (FIR) of MPDL3280A (anti-PDL1) in PD-L1-selected patients with non-small cell lung cancer (NSCLC). J Clin Oncol 2015;33(suppl); abstr 8028.

[64] Spira A, et al. Efficacy, safety and predictive biomarker results from a randomized phase II study comparing MPDL3280A vs docetaxel in 2L/3L NSCLC (POPLAR). J Clin Oncol 2015;33(suppl); abstr 8010.

[65] Damrauer JS, et al. Intrinsic subtypes of high-grade bladder cancer reflect the hallmarks of breast cancer biology. Proc Natl Acad Sci U S A 2014;111:3110-5.

[66] Gatalica Z, et al. Programmed cell death 1 (PD-1) and its ligand (PD-L1) in common cancers and their correlation with molecular cancer type. Cancer Epidemiol Biomarkers Prev 2014;23:2965-70. 
[67] Rosenberg SA, et al. Observations on the systemic administration of autologous lymphokine-activated killer cells and recombinant interleukin-2 to patients with metastatic cancer. N Engl J Med 1985;313:1485-92.

[68] Escudier B, et al. Axitinib versus sorafenib in advanced renal cell carcinoma: Subanalyses by prior therapy from a randomised phase III trial. Br J Cancer 2014;110:2821-8.

[69] Witte RS, et al. Eastern Cooperative Oncology Group phase II trial of ifosfamide in the treatment of previously treated advanced urothelial carcinoma. J Clin Oncol 1997;15:589-93.

[70] Lorusso V, et al. A phase II study of gemcitabine in patients with transitional cell carcinoma of the urinary tract previously treated with platinum. Italian Co-operative Group on Bladder Cancer. Eur J Cancer 1998;34:1208-12.

[71] Bambury RM, et al. The safety and efficacy of single-agent pemetrexed in platinum-resistant advanced urothelial carcinoma: A large single-institution experience. Oncologist 2015;20:508-15.
[72] Gallagher DJ, et al. Phase II study of sunitinib in patients with metastatic urothelial cancer. J Clin Oncol 2010;28:1373-9.

[73] Wulfing C, et al. A single-arm, multicenter, open-label phase 2 study of lapatinib as the second-line treatment of patients with locally advanced or metastatic transitional cell carcinoma. Cancer 2009;115:2881-90.

[74] Nguyen LT, Ohashi PS. Clinical blockade of PD1 and LAG3potential mechanisms of action. Nat Rev Immunol 2015; 15:45-56.

[75] Rizvi N, et al. Optimizing PD-L1 as a biomarker of response with pembrolizumab (pembro; MK-3475) as first-line therapy for PD-L1-positive metastatic non-small cell lung cancer (NSCLC): Updated data from KEYNOTE-001. J Clin Oncol 2015;33(suppl); abstr 8026.

[76] Shankaran V, et al. Correlation of gene expression signatures and clinical outcomes in patients with advanced gastric cancer treated with pembrolizumab (MK-3475). J Clin Oncol 2015;33(suppl); abstr 3026. 